\title{
Homöopathische Wirkstoffe in niederer Potenz: Immunpharmakologische in-vitro-Untersuchungen am menschlichen Immunsystem
}

\author{
Manfred W. Schmolz \\ EDI (Experimental and Diagnostic Immunology) $\mathrm{GmbH}$, DE-Reutlingen
}

$\mathrm{N}$ ach Mitteilung des Bundesgesundheitsministeriums (Stand 2000) gibt es in Deutschland 4'490 Homöopathen; weitere 6’000 Ärzte nutzen in ihrer Praxis antroposophische Methoden. Epidemiologischen Untersuchungen zufolge setzen etwa zwei Drittel aller niedergelassenen Ärzte zumindest zeitweise homöopathische Arzneimittel in ihrer Praxis ein [1,2]. Allein der Wirkmechanismus der homöopathischen Arzneimittel gibt den Wissenschaftlern nach wie vor Rätsel auf, und gegensätzliche Auffassungen zur klinischen Wirksamkeit wurden berichtet [3,4,5]. Einige Wissenschaftler gehen davon aus, dass ein möglicher Zugang zu einem - wie auch immer gearteten - Wirkmechanismus am ehesten auf der Basis physikalischer Modelle $\mathrm{zu}$ erreichen ist [6,7, 8,9]. Um Wirkmechanismen auf der Basis biochemischer, pharmakologischer oder allgemein materieller Grundprinzipien zu postulieren, sind Befunde hilfreich, die mit wissenschaftlich objektivierbaren Methoden Veränderungen im Organismus nachweisen, die in unmittelbarem Zusammenhang mit der Verabreichung von Homöopathika stehen. Der im Folgenden beschriebene Versuchsansatz war deshalb dazu gedacht, ein Modell aufzuzeigen, um potentielle regulative Effekte unterschiedlicher homöopathisch wirksamer Substanzen unter objektivierbaren Bedingungen zu untersuchen. Hierfür bot sich das menschliche Immunsystem aus mehreren Gründen an. Zum einen hat das Immunsystem mit seinen zahlreichen Botenstoffen speziell im Falle entzündlicher Erkrankungen an verschiedens-
Hintergrund: Hinsichtlich allgemein nachvollziehbarer Modelle, wie die Wirkungen homöopathischer Arzneimittel, vor allem in hohen Dilutionen, erklärt werden könnten, herrscht nach wie vor Unklarheit. Denn unmittelbare Veränderungen im Organismus direkt nach Verabreichung homöopathischer Arzneimittel lassen sich in der Regel nur schwer nachweisen. Fragestellung: Mit dem menschlichen Immunsystem als Zielorgan wurde daher in einem organotypischen Kulturmodell untersucht, ob zwei verschiedene homöopathische Komplexmittel sowie deren Einzelbestandteile im Sinne Hahnemanns in der Lage sind, die Synthese von immunregulativen Mediatoren anzuregen. Studien-Design: Orientierende Pilotstudie mit gesunden Probanden. Material und Methoden: Als optimales Surrogat für das menschliche Immunsystem wurden Vollblutkulturen verwendet, von 3 Spendern gewonnen. Vollblutkulturen jedes Spenders wurden mit 5 Konzentrationsstufen der Kombinationspräparate bzw. 5 Potenzstufen der jeweiligen Einzelmittel für 6 h vorinkubiert und anschliessend mit physiologischen Stimulanzien co-stimuliert, um die Synthese und Freisetzung von charakteristischen Mediatoren des unspezifischen (Tumor-Nekrose-Faktor alpha, (TNF- $\alpha$ ), Interleukin-10, (IL-10) und des spezifischen Immunsystems (Interleukin-5 (IL-5) und Interferon gamma, (IFN- $\gamma$ )) anzuregen. Die Messung der Mediatoren erfolgte anschliessend im Zellkulturüberstand mittels spezifischer ELISA Tests. Ergebnisse und Schlussfolgerungen: Sowohl mit den beiden hömopathischen Komplexmitteln als auch mit deren Einzelkomponenten ergaben sich individualspezifisch unterschiedliche Effekte auf die Freisetzung der genannten Mediatoren. Aufgrund der geringen Fallzahl von nur 3 Spendern kann dies nur als Hinweis darauf gewertet werden, dass die homöopathischen Substanzen unterschiedliche regulatorische Ebenen im menschlichen Immunsystem beeinflussen könnten.

Schlüsselwörter: Homöopathie, Immunsystem, Immunregulation, Zytokine

\section{Homeopathic Drugs at Low Dilutions:}

\section{Immunopharmacological In-vitro Investigations in the Human Immune System}

Background: Concerning generalized models able to explain the mechanisms of action of homeopathic drugs at low dilutions, there is still uncertainty because it is quite difficult to demonstrate acute changes in a human organism in response to homeopathic therapy. Aim of the Study: Using the human immune system as a target organ, an organotypic culture model served to determine whether two different homeopathic combination preparations together with their individual components would be capable to induce the synthesis of immunoregulatory mediators. Design of the Study: Pilot study with healthy volunteers. Methods: Human whole-blood cultures, being an optimal surrogate for the human immune system, were prepared from 3 donors. Whole blood cultures from each donor were preincubated for $6 \mathrm{~h}$ with 5 different dilutions of the homeopathic combination preparations, and 5 potencies of the respective individual components of each combination preparation, followed by the costimulation with physiologic stimuli able to specifically induce mediators from the nonspecific immune system (Tumor Necrosis Factor alpha (TNF- $\alpha$ ), Interleukin-10, (IL-10)) and the specific immune system (Interleukin5 (IL-5) and Interferon gamma (IFN- $\gamma$ )). Thereafter, mediators released were determined in the cell culture supernatant with specific ELISA tests. Results and Conclusion: Both homeopathic combination preparations as well as their individual components displayed differential individual effects on the release of the mediators tested. Because only 3 blood donors were used in this study, these data may be regarded as a trend showing that homeopathic drugs might affect regulatory pathways in the immune system also under ex vivo conditions.

Key Words: Homeopathy, immune system, immunoregulation, cytokines 
ten Organsystemen eine erhebliche (patho-)physiologische Bedeutung [10]. Zum anderen tragen eine Fülle regulatorischer Interaktionen von Immunzellen untereinander dazu bei, akute Entzündungsprozesse nicht nur zu initiieren, sonden auch zu begrenzen. Basierend auf früheren Publikationen [11] ging es im vorliegenden Versuchsansatz vor allem darum, grundsätzlich etwaige Veränderungen von Immunparametern aufzuzeigen, wenn lebende Zellen in vitro mit homöopathisch wirksamen Substanzen in niedrigen Potenzen in Berührung kommen. Das verwendete organotypische Kulturmodell „Vollblut“ vereinigte dabei sowohl die Forderung nach Kulturbedingungen grösstmöglicher Übereinstimmung mit den Verhältnissen in vivo als auch jene nach objektiven Grundbedingungen, mittels welcher die Entstehung reiner Placebo-Wirkungen ausgeschlossen werden können. Untersucht wurden zwei verschiedene homöopathische Komplexmittel - Angina-Gastreu und GrippeGastreu (Dr. Reckeweg \& Co GmbH, Bensheim) sowie deren Einzelsubstanzen. Aufgrund der immensen Vielfalt regulatorischer Aktivitäten [12,13] des menschlichen Immunsystems wurde in dieser orientierenden Pilotuntersuchung das Augenmerk auf einige zentrale Marker des unspezifischen und des spezifischen Immunsystems gelenkt: TNF- $\alpha$, IL-10, IFN- $\gamma$ und IL-5. Ihre wesentlichen Aufgaben im Immunsystem verdeutlicht Tabelle 1.

\section{Material und Methoden}

\section{Beschreibung der Prüfsubstanzen}

Die homöopathischen Einzelsubstanzen sowie die beiden untersuchten homöopathischen Komplexmittel wurden von der Dr. Reckeweg GmbH (Bensheim) zur Verfügung gestellt. Beide Präparate haben in Deutschland den Status eines homöopathischen Arzneimittels. Die Komplexmittel waren wie folgt zusammengesetzt:

Angina-Gastreu $^{\circledR}$ S R1 Tropfen: 10g enthalten: Apis mellifica D4 1g, Atropa belladonna D4 1g, Calcium iodatum D4 1g, Hepar Sulfuris D12 1g, Kalium bichromicum D4 0,01g, Lachesis muta

Tab. 1. Schlüsselfunktionen der ausgewählten Immunparameter im Immunsystem

\begin{tabular}{|c|c|}
\hline Parameter & Funktionelle Bedeutung \\
\hline $\begin{array}{l}\text { Tumornekrose-Faktor } \alpha \\
(\text { TNF- } \alpha)\end{array}$ & $\begin{array}{l}\text { Ein Schlüsselmediator bei der Abwehr bakterieller Infek- } \\
\text { tionen, allgemein pro-inflammatorisch wirkendes Cytokin, } \\
\text { wird sehr früh in Abwehrreaktionen hauptsächlich von } \\
\text { Monozyten freigesetzt. }\end{array}$ \\
\hline Interleukin-10 (IL-10) & $\begin{array}{l}\text { Vorwiegend von Monozyten und B-Lymphozyten gebildetes } \\
\text { Cytokin, inhibiert Cytokin-Synthesen von Monozyten/Ma- } \\
\text { krophagen, hemmt z.B. die Antigen-Präsentation. }\end{array}$ \\
\hline Interferon- $\gamma($ IFN- $\gamma)$ & $\begin{array}{l}\text { Bedeutender Mediator aus dem spezifischen Immunsystem } \\
\text { (T-Helferzellen, Typ 1). Stellt eine wichtige Verbindung zwi- } \\
\text { schen dem spezifischen und unspezifischen Immunsystem } \\
\text { her, wie z.B. Antigenpräsentation und Aktivierung von Ma- } \\
\text { krophagen. }\end{array}$ \\
\hline Interleukin-5 (IL-5) & $\begin{array}{l}\text { Charakteristischer Mediator aus dem spezifischen Immun- } \\
\text { system (T-Helferzellen, Typ 2), zusammen mit TGF- } \beta \text { Induk- } \\
\text { tion der IgA-Synthese, Wachstumsfaktor für B-Lymphozyten } \\
\text { aber auch Aktivator für eosinophile Granulozyten. }\end{array}$ \\
\hline
\end{tabular}

Tab. 2. Bezeichnungen der Testsubstanzen mit eingesetzten Konzentrationen

\begin{tabular}{|c|c|c|}
\hline Bezeichnung & Charge & $\begin{array}{l}\text { Verdünnungsstufen } \\
\text { (Endkonz. pro ml Kultur) }\end{array}$ \\
\hline Apis & 0002R0108 bis -112 & D2, D3, D4, D5, D6 \\
\hline Belladonna & $0002 R 0114$ bis -118 & D2, D3, D4, D5, D6 \\
\hline Calcium iodatum & $0002 R 0124$ bis -128 & D2, D3, D4, D5, D6 \\
\hline Hepar sulfuris & $0002 R 0131$ bis -135 & D10, D11, D12, D13, D14 \\
\hline Kalium bichromicum & $9902 R 0024$ bis -026 & $\mathrm{D} 2, \mathrm{D} 3, \mathrm{D} 4$ \\
\hline Kalium bichromicum & $0002 R 0139$ bis -140 & D5, D6 \\
\hline Lachesis & $0002 R 0154$ bis -158 & D10, D11, D12, D13, D14 \\
\hline Marum verum & $0002 R 0166$ bis -170 & D4, D5, D6, D7, D8 \\
\hline Mercurius subl. corr. & $0002 R 0171$ bis -175 & D3, D4, D5, D6, D7 \\
\hline Phytolacca & $0002 R 0187$ bis $-190,-193$ & D2, D3, D4, D5, D6 \\
\hline R1 Angina-Gastreu ${ }^{\circledR}$ & 002R0253 & $\begin{array}{l}1: 50,1: 100,1: 200,1: 400 \\
1: 800 \text { (C1 - C5) }\end{array}$ \\
\hline Aconitum & $0002 R 0196$ bis -200 & D2, D3, D4, D5, D6 \\
\hline Baptisia & $0002 R 0209$ bis -213 & D2, D3, D4, D5, D6 \\
\hline Bryonia & $0002 R 0222$ bis -226 & D2, D3, D4, D5, D6 \\
\hline Camphora & $0002 R 0231$ bis -235 & D1, D2, D3, D4, D5 \\
\hline Causticum Hahnemanni & $0002 R 0243$ bis -247 & D4, D5, D6, D7, D8 \\
\hline Eucalyptus & $17118,0002 \mathrm{R} 0436$ bis -439 & $\mathrm{D} 1=\Phi, \mathrm{D} 2, \mathrm{D} 3, \mathrm{D} 4, \mathrm{D} 5$ \\
\hline Eupatorium perfoliatum & $0002 R 0520$ bis -524 & D1, D2, D3, D4, D5 \\
\hline Ferrum phoshoricum & $0002 R 0262,-264$ bis -267 & D6, D7, D8, D9, D10 \\
\hline Gelsemium & $0002 R 0278$ bis -282 & D4, D5, D6, D7, D8 \\
\hline Sabadilla & $0002 R 0283$ bis -287 & D4, D5, D6, D7, D8 \\
\hline R6 Grippe-Gastreu ${ }^{\circledR}$ & 0002R0255 & $\begin{array}{l}1: 50,1: 100,1: 200,1: 400 \\
1: 800 \text { (C1 - C5) }\end{array}$ \\
\hline Ethanol $30 \%$ & $0001 R 0600$ & eingesetzt: 1:100 \\
\hline Ethanol $50 \%$ & $0001 R 0043$ & eingesetzt: 1:100 \\
\hline Ethanol $70 \%$ & $0001 R 0662$ & eingesetzt: 1:100 \\
\hline Ethanol $90 \%$ & $0001 R 0086$ & eingesetzt: 1:100 \\
\hline
\end{tabular}

D12 1g, Marum verum D6 1g, Mercurius sublimatus corrosivus D5 $1 \mathrm{~g}$, Phytolacca D4 1g, Ethanol, Aqua purificata. Enthält 38 Vol.-\% Alkohol,
Anwendung: Die Anwendungsgebiete leiten sich von den homöopathischen Arzneimittelbildern ab. Dazu gehören: Besserung der Beschwerden bei aku- 
ten Infekten der oberen Luftwege und des Rachenraums.

Grippe-Gastreu ${ }^{\circledR}$ S R6 Tropfen; Zusammensetzung: $10 \mathrm{ml}$ enthalten: Aconitum napellus D4 1ml, Baptisia D4 1ml, Bryonia D4 1ml, Camphora D3 1ml, Causticum Hahnemanni D6 1ml, Eucalyptus D3 $1 \mathrm{ml}$, Eupatorium perfoliatum D3 1ml, Ferrum phosphoricum D8 1ml, Sabadilla D6 $1 \mathrm{ml}$, Gelsemium D6 1ml, Ethanol, Aqua purificata. Enthält 41 Vol.-\% Alkohol. Anwendung: Die Anwendungsgebiete leiten sich von den homöopathischen Arzneimittelbildern ab. Dazu gehören: Grippale Infekte (Erkältungskrankheiten).

\section{Herstellung der Vollblutkultur}

Vollblutkulturen wurden modifiziert nach der Methode von MEYAARD et al. hergestellt [14]. Es wurde hierfür frisch entnommenes peripheres Blut verwendet. Die Blutabnahme erfolgte mittels einer 20-ml-Spritze, befüllt mit Natrium-Heparin (50 IU/ml Blut, Ratiopharm Deutschland). Nach Verdünnung (1:2) in physiologischem Hanks Puffer (Hanks Balanced Salt Solution HBSS, Fa. Biochrom KG, Deutschland) erfolgte die Aussaat in Mikrotiterplatten (96 Loch, Fa. Greiner Deutschland, $150 \mu \mathrm{l} /$ Loch). Für jede zu untersuchende Substanz wurde stets das Blut von 3 verschiedenen Spendern herangezogen, um mögliche interindividuelle Schwankungen in der Reaktion auf die Testsubstanzen sichtbar zu machen. Unmittelbar nach Aussaat in die Zellkulturplatten wurden die homöopathischen Substanzen bzw. die beiden homöopathischen Komplexmittel in verschiedenen Verdünnungen hinzugegeben (dargestellt in Tab. 2).

\section{Vorbehandlung mit den homöo- pathischen Substanzen/Komplex- mitteln}

Die Testsubstanzen wurden in jeweils 5 verschiedenen Konzentrationen (Kombinationspräparate) bzw. 5 Potenzstufen (Einzelmittel) eingesetzt. Jede Kultur/Substanz wurde als Dreifachwert angelegt. Als Potenz-Reihe wurde jeweils die im entsprechenden Medikament enthaltene Potenzstufe sowie die beiden darunter und darüber liegenden Dezimalpotenzen eingesetzt. Bis auf die Verdünnungsstufen der

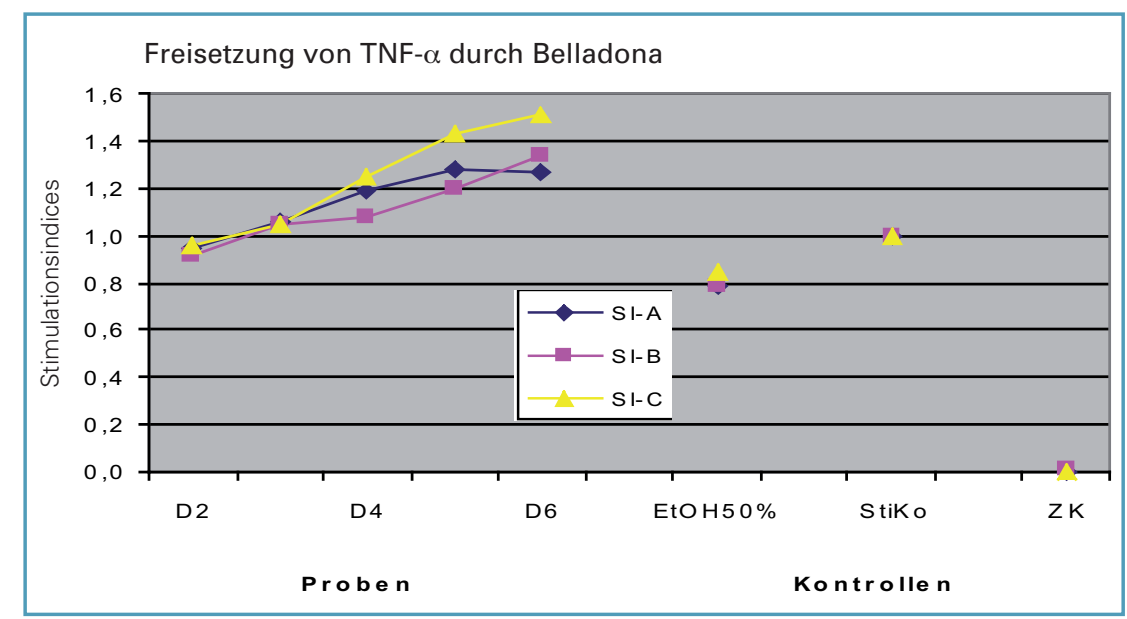

Abb. 1. Freisetzung von TNF- $\alpha$ durch verschiedene Dilutionen von Belladonna.

Zu Abb. 1-7: Vollblutleukozyten-Kulturen wurden mit den homöopathischen Testsubstanzen bzw. den homöopathischen Komplexmitteln in den in Tabelle 2 gezeigten Dilutionen $6 \mathrm{~h}$ vorinkubiert und die Freisetzung der jeweiligen Mediatoren mit den in Material und Methoden genannten Stimulanzien induziert. Dargestellt sind die Resultate der aus den Kulturüberständen bestimmten Mediatoren, berechnet in Form der Stimulationsindices (SIWerte) von Vollblutleukozytenkulturen dreier verschiedener Spender (A bis C). EtOH = Ethanolkontrolle der Konzentrationen, stimuliert; StK = Stimuluskontrolle (Pufferkontrolle mit Stimulanz, aber ohne Prüfsubstanzen), ZK=Zellkontrolle (= Negativkontrolle, unstimulierte Kultur mit Puffer).

Gesamtpräparate wurden alle Proben vom Auftraggeber vorgefertigt angeliefert. Die Vorverdünnungen der Proben sowie die Verdünnungsstufen der Komplexmittel wurden mit Kulturmedium (RPMI 1640, Fa. Biochrom) mit 5\% autologem Serum (als Lösungsvermittler) hergestellt. Als Kontrollen diente Ethanollösung in den Konzentrationen der Probensubstanzen. Um sicherzustellen, dass Ethanol-Effekte sich auf ein Minimum beschränkten, war eine generelle Vorverdünnung aller Proben bzw. der Kontroll-Lösungen von 1:100 (bezogen auf die Endkonzentration in der Kultur) erforderlich. Nach Zugabe aller Testsubstanzen wurden die Vollblutkulturen $6 \mathrm{~h}$ mit den Testsubstanzen im Brutschrank vorinkubiert.

\section{Ko-Stimulation der Vollblutkulturen zur Mediatorensynthese}

Nach 6h Vorinkubation der Vollblutkulturen mit den homöopathischen Wirkstoffen erfolgte die Ko-Stimulation mit Monozyten-spezifischen und T-Zell-spezifischen Aktivatoren. Mit AB Plasma wurde nach der Methode von CREE und BLAIR [15] opsoniertes Zymosan (= partikuläres und damit phagozytierbares Zellwandmaterial aus Hefen, geliefert von Sigma-Aldrich,
Deutschland) hergestellt und in einer Endkonzentration von $1 \mathrm{mg} / \mathrm{ml}$ eingesetzt (Anregung der TNF- $\alpha$ - und IL-10Synthese). Die Stimulierung der Lymphokine erfolgte durch Aktivierung der Zellen mit PHA (IFN- $\gamma$ ), bzw. mit PHA + anti-CD28 Antikörpern (für IL-5). Anti-CD28 Antikörper stammten von R\&D Systems (Endkonzentration 300 ng/mL), PHA (Biochrom KG) wurde in einer Endkonzentration von 1,25 $\mu \mathrm{g} / \mathrm{ml}$ verwendet. Nach 24 h (Monokine IL-10, TNF- $\alpha$ ) bzw. 48 h (Lymphokine IFN- $\gamma$, IL-5) wurden die Überstände durch Zentrifugieren der Platten $(10 \mathrm{~min}$, $600 g)$ geerntet und bis zur Messung mit spezifischen ELISA Tests (R\&D Biosystems) bei $-20{ }^{\circ} \mathrm{C}$ eingefroren.

\section{Mediatoren-Bestimmung}

Die Quantifizierung der Mediatoren im Kulturüberstand erfolgte mittels klassischer Sandwich-ELISAs. (R\&D Biosystems, Heidelberg). Alle ELISA wurden den Angaben des Herstellers zufolge durchgeführt.

\section{Auswertung}

Verschiedene Spender weisen in der Regel deutlich unterschiedliche Synthese-Kapazitäten bezüglich der gemessenen Mediatoren auf [16], was den Vergleich der Resultate zwischen 


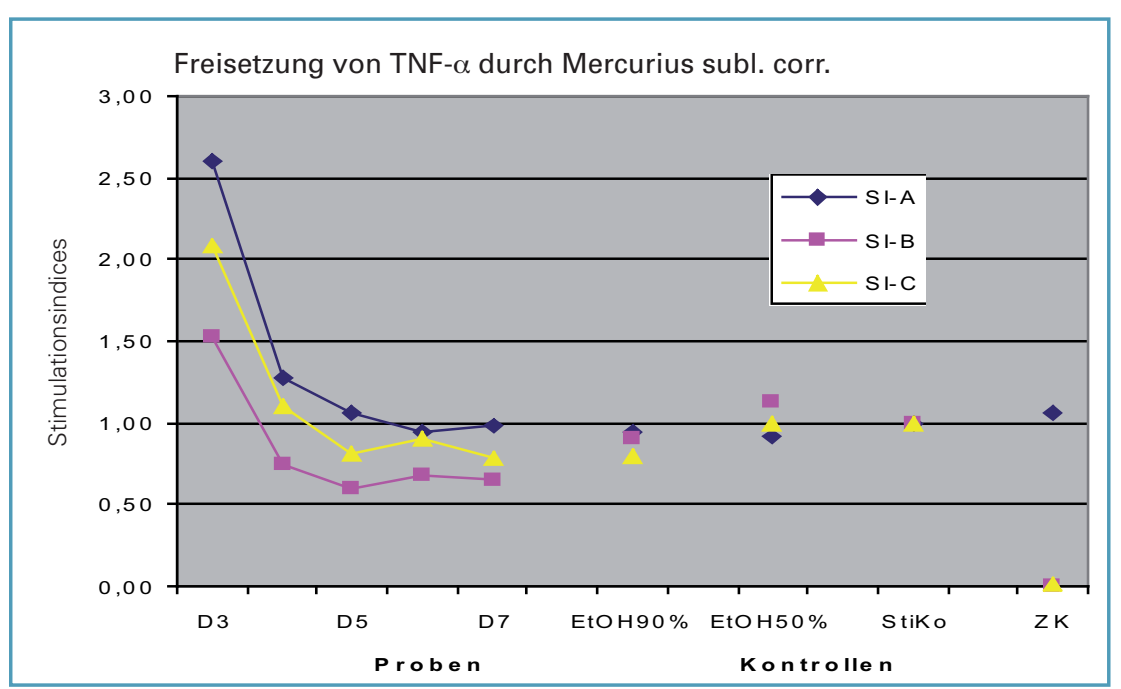

Abb. 2. : Freisetzung von TNF- $\alpha$ durch verschiedene Dilutionen von Mercurius subl. corr.

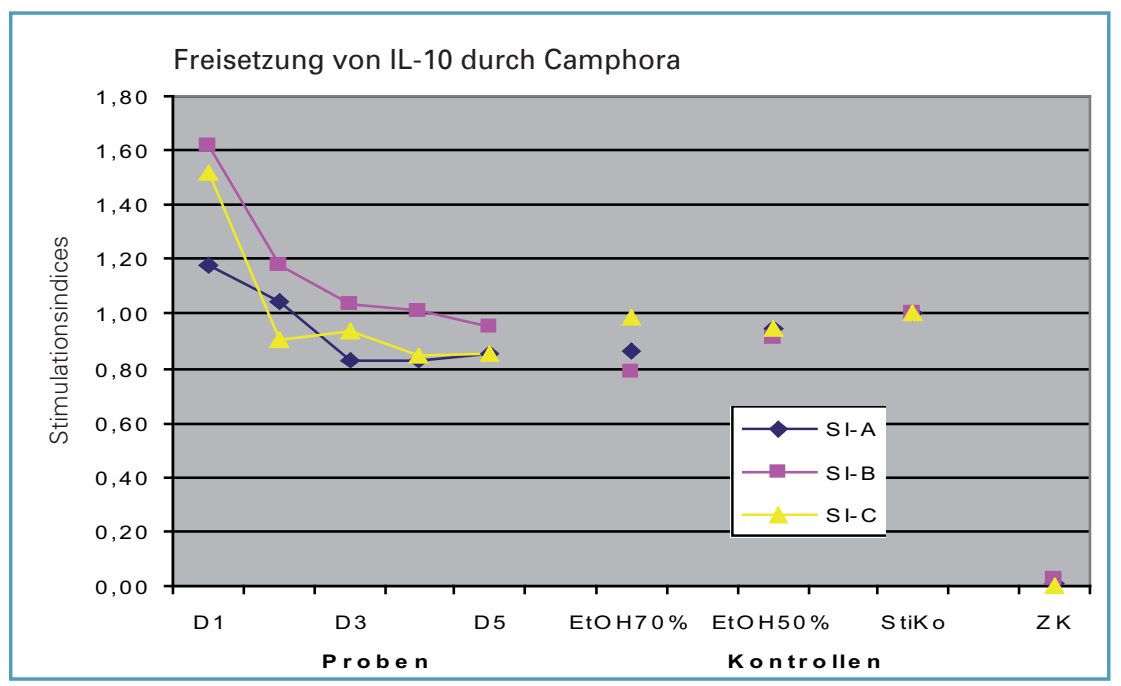

Abb. 3. Freisetzung von IL-10 durch verschiedene Dilutionen von Camphora.

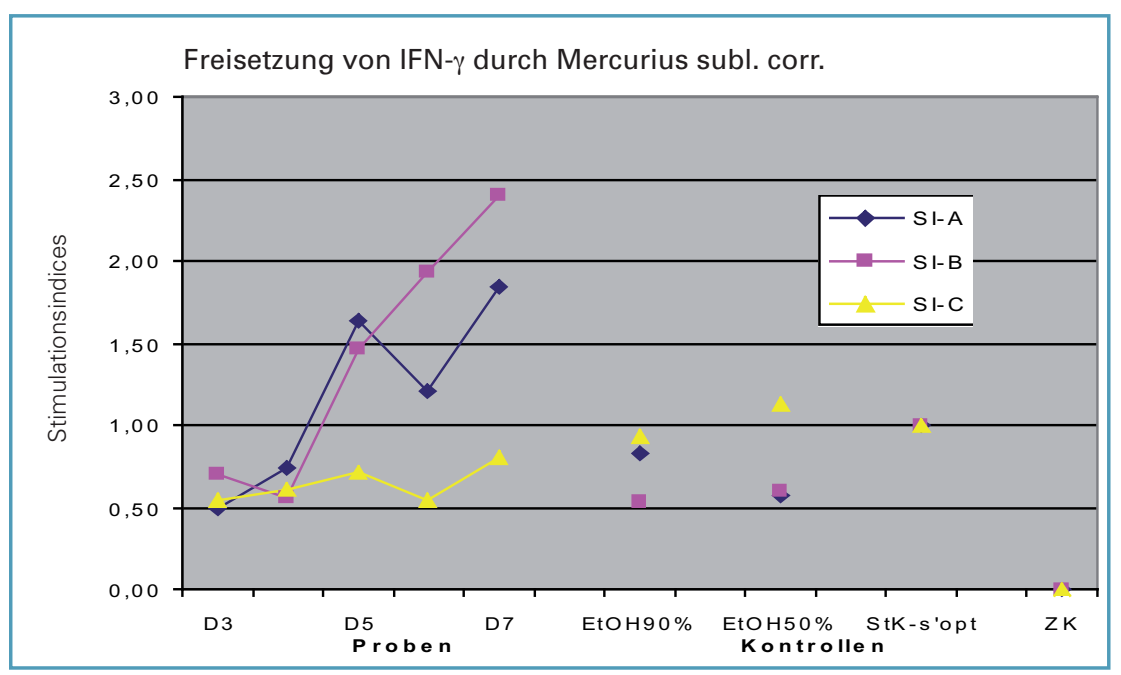

Abb. 4. Freisetzung von Interferon- $\gamma$ durch verschiedene Dilutionen von Mercurius subl. corr. den einzelnen Spendern erschwert. Deshalb wurden die absoluten Messwerte in Stimulationsindizes umgerechnet [17]. Dadurch lassen sich die Steigerungen oder Verminderungen in der Freisetzung der jeweiligen Botenstoffe als Vielfaches der Kontrolle darstellen, was die Vergleichbarkeit der Werte deutlich verbessert. Aufgrund langjähriger Erfahrungen mit dem Kultursystem und dessen biologischen Schwankungsbreiten wurden als biologisch relevante Effekte jene eingestuft, die in der Stimulation einen Stimulationsindex von 1,3 erreichten oder überstiegen bzw. als Inhibition 0,7 erreichten oder überschritten.

Berechnung Stimulationsindex:

$$
\frac{\text { Probenwert }}{\text { Stimulationskontrolle }}
$$

wobei jeweils diejenigen Ethanol-Kontrollen in die Formel eingesetzt wurden, die in der Konzentration den entsprechenden Verdünnungsstufen der Proben entsprachen.

\section{Ergebnisse}

Nur wenige Substanzen waren insgesamt bei der Testung auf Elastase aktiv (Daten nicht gezeigt).

\section{TNF-alpha}

Exemplarisch für die Beeinflussung der TNF- $\alpha$-Synthese durch die Einzelsubstanzen sind zwei typische Effekte in Abb. 1 und Abb. 2 dargestellt. Sie verdeutlichen, dass TNF- $\alpha$ in ganz unterschiedlicher Weise beeinflusst wurde. Mit Belladonna (Abb. 1) ergab sich eine nahezu spenderunabhängige Stimulation der TNF- $\alpha$-Synthese, mit dem Maximum des Effektes in hohen Dilutionen und klar konzentrationsabhängigen Verläufen. Mercurius subl. corr. hingegen zeigte spenderabhängig mit niedrigen Dilutionen wie D3 (=hohe Konzentration) eine bis auf das 2,5fach gesteigerte TNF- $\alpha$-Synthese (Abb. 2), die mit hohen Dilutionen wie D7 (geringe Konzentration) bei einem Spender sogar in eine Inhibition mündete. Von der Stärke der Effekte und einem Verlauf ähnlich dem von Belladonna sind ferner Eupatorium, Eucalyptus, Bryonia, Lachesis und Phyto- 


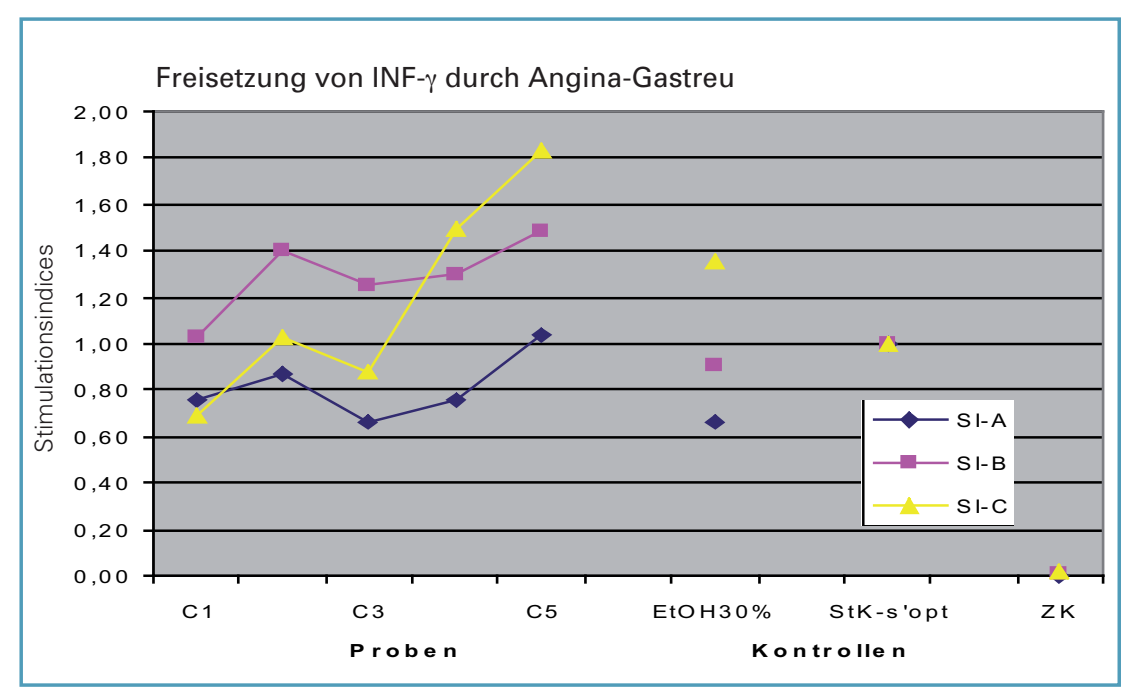

Abb. 5. Freisetzung von Interferon- $\gamma$ durch das Gesamtpräparat Angina-Gastreu.

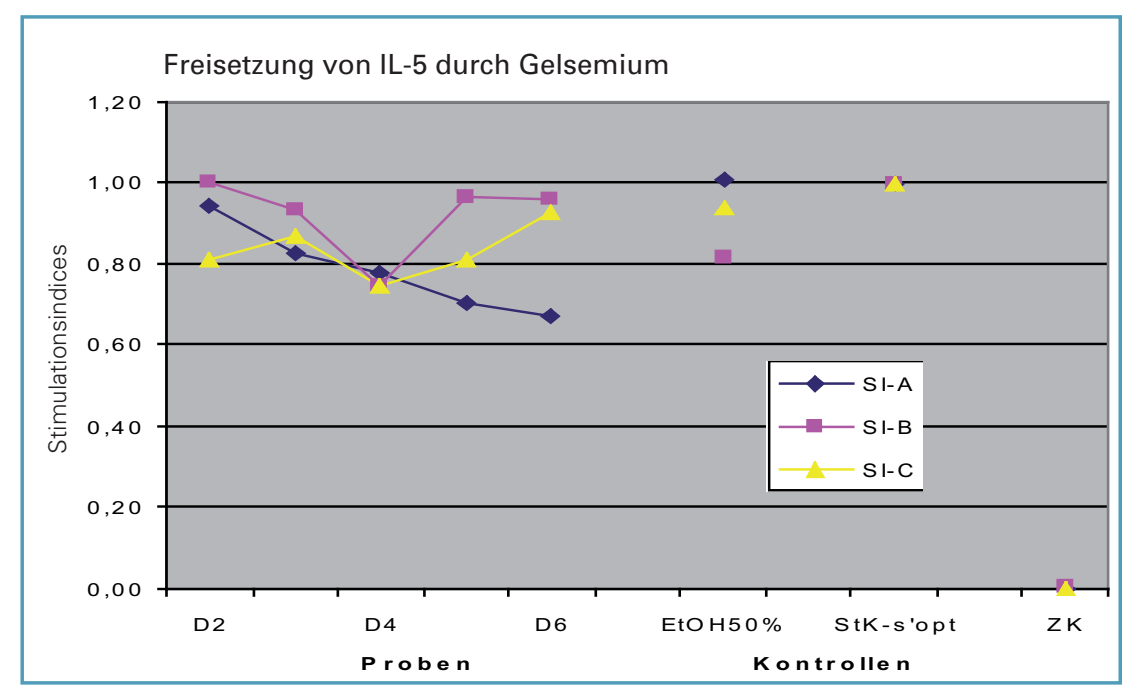

Abb. 6. Freisetzung von Interleukin-5 durch verschiedene Dilutionen von Gelsemium.

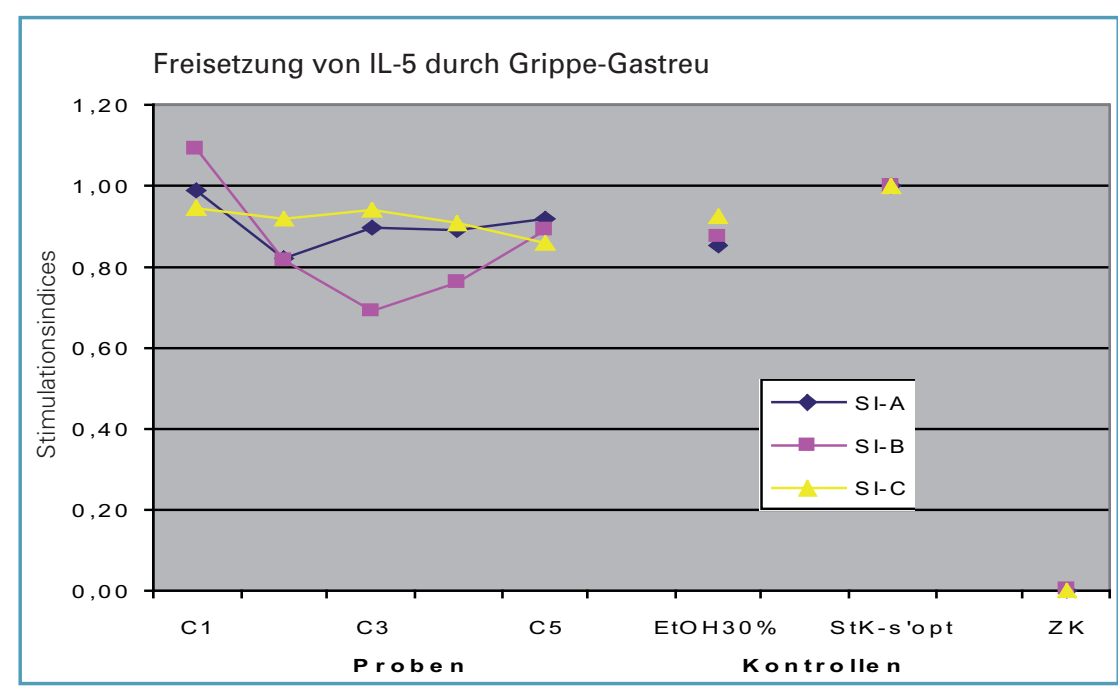

Abb. 7. Freisetzung von IL-5 durch das Gesamtpräparat Grippe-Gastreu. lacca hervorzuheben. Die Mehrzahl der anderen Einzelmittel (u.a. Apis, Hepar, Aconitum, Baptisia, Causticum, Gelsemium) erwies sich als deutlich schwächer aktiv (SI-Werte im Falle einer Stimulation kaum über 1,2).

\section{Interleukin-10}

Interleukin-10 wurde von mehreren Einzelmitteln in niedrigen Dilutionen mässig stimuliert. Ähnliche Kurvenverläufe wie die in Abb. 3 exemplarisch dargestellte Synthese von IL-10 mit unterschiedlichen Dilutionen von Camphora ergaben sich darüberhinaus auch mit Eucalyptus, Phytolacca und Mercurius. Wiederum war Mercurius subl. corr. eines der stärksten Einzelmittel bzgl. der Stimulation der IL-10-Synthese in niedrigen Dilutionen (Effektkurven ähnlich wie bei TNF- $\alpha$ in Abb. 2). Andererseits konnten auch spenderabhängig leichte Inhibitionen der IL-10-Synthesen beobachtet werden (z.B. mit Hepar, Gelsemium, Causticum, Sabadilla oder Ferrum phosphoricum). Für die Mehrzahl der restlichen Einzelmittel waren mit IL-10 monotone Potenz-Effekt-Kurven zu sehen, die sich jedoch im Bereich von SI-Werten von 0,8 bis 1,2 abspielten. Das Gleiche galt für die beiden homöopathischen Komplexmittel AnginaGastreu und Grippe-Gastreu.

\section{Interferon gamma}

Hinsichtlich der Effekte der homöopathischen Substanzen auf das spezifische Immunsystem konnten deutlich mehr Substanzen identifiziert werden, die regulative Effekte - in Abb. 4 mit IFN- $\gamma$ als Messgrösse - zeigten. Kurvenverläufe wie für Mercurius subl. corr. dargestellt (Abb. 4), ergaben sich in ähnlicher Form auch für Hepar, Eucalyptus, Camphora und Eupatorium. Einzelmittel, für die eine anfängliche Stimulation in niedrigen Dilutionen in eine tendenzielle Inhibition mündete, waren u.a. Gelsemium, Causticum und Sabadilla. Substanzen mit inhibitorischer Wirkung über mehrere Dilutionen waren spenderabhängig Marum, Phytolacca, Baptisia, Bryonia und Aconitum.

In Abb. 5 ist der Einfluss des homöopathischen Komplexmittels Angina-Gastreu auf die Freisetzung von 
IFN- $\gamma$ dargestellt. Auch hier ist das individuelle Ansprechen der Spenderleukozyten auf das homöopathische Komplexmittel deutlich zu erkennen, worauf in der Diskussion noch näher eingegangen wird.

\section{Interleukin-5}

Unter den homöpathischen Substanzen mit Einfluss auf die Synthese von IL-5 (vergl. Abb. 6, Wirkung von Gelsemium) fanden sich wesentlich mehr inhibitorische als stimulierende. Dieser Effekt korrespondiert sehr gut mit den Ergebnissen zu IFN- $\gamma$, wo sehr viele Einzelmittel mit stimulierenden Effekten gefunden wurden. Th1-Zellen werden in ihrer Funktion als Produzenten von IFN- $\gamma$ als direkte regulatorische Gegenspieler der Th2-Zellen (Synthese von IL-5) gesehen. Abb. 7 stellt die Effekte des homöpathischen Komplexmittels Grippe-Gastreu auf die IL-5-Synthese dar. Vor allen Dingen in hohen Dilutionen (1:200 bis 1:800) war ein inhibitorischer Einfluss auf die IL-5 Freisetzung zu beobachten.

\section{Diskussion}

Die hier durchgeführten immunpharmakologischen in-vitro-Untersuchungen mit homöopathischen Wirkstoffen sowie zwei homöopathischen Komplexmitteln zeigen, dass viele der getesteten homöopathischen Substanzen im Vollblut-System eine Aktivität entfalteten, die aufgrund der geringen Anzahl an Blutspendern momentan jedoch nur als Trend gewertet werden kann. Dennoch ist vom Profil der bestimmten Mediatoren her zu erkennen, dass womöglich beide Bereiche des Immunsystems, das unspezifische mit den Phagozyten als Indikatorzellen und das spezifische (hier T-Lymphozyten) von den homöopathischen Wirkstoffen in unterschiedlichen Wirkrichtungen angesprochen wurden. Dabei hat sich die empirische Erfahrung erneut bestätigt, dass immunpharmakologische Aktivitäten von natürlichen Wirkstoffen bzw. Zubereitungen ausgeprägte interindividuelle Wirkunterschiede besitzen. Auf dieses wesentliche Charakteristikum wird im Folgenden noch näher eingegangen.

\section{Zusammenfassende Beurteilung der Wirkungen homöopathischer Substanzen auf das unspezifische Immunsystem}

Mit den beiden Markern TNF- $\alpha$ und IL-10 für das unspezifische Immunsystem konnten sowohl inhibitorische als auch stimulatorische Einflüsse der homöopathischen Wirkstoffe festgestellt werden. Die Beobachtung von spenderabhängigen und spenderunabhängigen, zudem oftmals ganz unterschiedlichen Wirkoptima einzelner homöopathischer Substanzen (vergl. z.B. Belladonna und Mercurius subl. corr.) ist ein anschauliches Beispiel für die eingangs erwähnte interindividuelle Aktivität von Naturstoffen am Immunsystem. Während z.B. Apis eine verstärkte TNF- $\alpha$-Synthese bei einem Wirkoptimum zwischen D3 und D4 induzierte, erreichte Mercurius subl. corr erst in hohen Konzentrationen (=niedrigste Dilution) das Maximum seines Effektes (2,5-fache der stimulierten Kontrolle). Interessanterweise schwenkte diese aktivierende Wirkung von Mercurius subl. corr. bei Leukozyten eines anderen Spenders in niedrigen Konzentrationen (=höhere Dilution) in eine Hemmung um. Derartige Dosis-Wirkungsbeziehungen sind meistens Ausdruck der Präsenz mehrerer Wirkstoffe mit unterschiedlichen Wirkweisen und Wirkoptima in unterschiedlichen Dosis-Bereichen. Weil Monozyten/Makrophagen wichtige regulatorische Bindeglieder zwischen unspezifischem und spezifischem Immunsystem darstellen [18], könnte hieraus auf eine Beeinflussung der Infektabwehr durch die untersuchten Wirkstoffe geschlossen werden, wenn diese vorläufigen Ergebnisse mit einem grösseren Spenderkollektiv reproduziert werden können. Die Effekte der meisten Substanzen auf die IL-10Synthesen sind insofern bemerkenswert, als sich nur in den wenigsten Fällen die typische antagonistische Rolle des Mediatorenpaares TNF- $\alpha / \mathrm{IL}$ $10[19,20]$ bestätigte. Normalerweise fungieren die Cytokine TNF- $\alpha$ und IL10 als Gegenspieler im Immunsystem. Sie können die Aktivitäten von THelferlymphozten oder Makrophagen hoch- und wieder herunterregulieren. So wurde die IL-10-Synthese beispiels- weise von Mercurius, Phytolacca, Eucalyptus und Camphora stimuliert (eindeutige Potenz/Verdünnungs-Abhängigkeit mit Maximum dieses Effektes in jeweils der niedrigsten Potenz/Verdünnung). Damit verliefen z.B. die Effekte der homöopathischen Substanzen Mercurius und Eucalyptus gleichsinnig auf die TNF- $\alpha$ - und IL-10Freisetzung (beides wurde stimuliert). Dieses Aktivitätsprofil war mit den beiden homöopathischen Komplexmitteln nicht gegeben.

\section{Wirkungen homöopathischer Substanzen am spezifischen Immunsystem}

Die bereits erwähnten besonderen Charakteristika immunpharmakologischer Wirkungen von Naturstoffen waren beim spezifischen Immunsystem noch tiefgreifender ausgeprägt. In diversen Ansätzen mit unterschiedlichen Proben ergaben sich exzellente stimulierende Wirkungen bei IFN- $\gamma$ um mehr als das Doppelte der stimulierten Kontroll-Kultur bei einzelnen Spendern mit Apis, Belladonna, Lachesis (D14!) und Mercurius (D7!) sowie knapp dem Doppelten bei etlichen anderen Einzelmitteln. Wirkungen wie z.B. die von Apis sind besonders hervorzuheben: Bei 2 Spendern zeigte sich ein nahezu paralleler Verlauf der Potenz-Effekt-Kurven, wobei jedoch die Kurve des einen praktisch ganz auf der stimulierenden Seite verlief, während bei dem anderen Spender alle Potenzstufen inhibierend wirkten. Erklärt wird diese Individualität der Reaktionen des menschlichen Immunsystems durch die personenspezifische Ontogenese des Abwehrsystems (u.a. bis dato durchgemachte Infektionen, individuelle Stresstoleranz, Spektrum täglich zugeführter Noxen), die das Verhaltensmuster der Leukozyten auch im Hinblick auf die Reaktionen gegenüber pharmakologisch aktiven Verbindungen mitbestimmen. In besonderem Masse gilt dies für Wirkstoffe wie Homöopathika, die kein "Alles-oder-Nichts-Signal" induzieren, wie es z.B. die klassischen Immunsuppressiva, Antiphlogistika oder gar Zytostatika bewirken. Milde wirksame Pharmaka lassen dem Immunsystem im Gegenteil die Möglichkeit, sich ver- 
ändernden Bedingungen nach wie vor flexibel anzupassen und situativ auf akute Notwendigkeiten $\mathrm{zu}$ reagieren. Man könnte dies als eine therapeutische Hilfe zur Optimierung verstehen, die bei jedem Patienten eine auf dessen Verhältnisse abgestimmte Nachregulation erlaubt, ohne eine bestimmte Richtung vorzugeben. Eine solche Wirkweise würde insgesamt relativ gut der homöopathischen Theorie im Hinblick auf die Summenwirkung vielfältiger und an verschiedenen Stellen des Körpers ansetzender Teilwirkungen, welche letztlich erst zusammengenommen eine "ganzheitliche" Gesamtwirkung zustandebringen, entsprechen [21]. Vergleichbare Vorgänge - wenn auch in einem anderen Kontext - konnten in früheren Untersuchungen an Vollblut beobachtet werden [22,23]. Es ist vorstellbar, dass ein Überwiegen stimulatorischer Wirkungen auf die IFN- $\gamma$-Synthese mit andererseits vermehrten Hemmungen im Bereich von IL-5 eine präferentielle Unterstützung klassischer Th1-Antworten induzieren könnte. Diese sind es auch, welche das Immunsystem üblicherweise zur Abwehr der meisten Infektionen einsetzt [24]. Im Vergleich zu den oben genannten homöopathischen Einzelmitteln fielen die Aktivitäten der beiden Komplexmittel insgesamt eher gering - oder besser gesagt widersprüchlich - aus. Beispielsweise bei der Freisetzung und Synthese von IFN- $\gamma$ zeigte R1 AnginaGastreu bei zwei Spendern eine Stimulation über $30 \%$, bei zwei anderen hingegen eine Hemmung von über $30 \%$. Für den gleichen Botenstoff konnte bei Grippe-Gastreu eine leichte Hemmung (zw. 20 und 30\%) in zwei und eine starke Hemmung ( $>30 \%$ ) in einem Fall dokumentiert werden (Daten nicht gezeigt). Der beobachtete Trend in den immunpharmakologischen Aktvitäten der hier getesteten homöopathischen Substanzen wäre aus regulations-medizinischer und immunologischer Sicht durchaus mit den medizinischen Einsatzgebieten der beiden homöopathischen Komplexmittel zu vereinbaren. Wie sich dieses interessante Konzept einer durch potenzierte homöopathische Substanzen gesteuerten Feinregulation immunolo- gischer Abwehrreaktionen - wesentlich bei der Entstehung und Therapie entzündlicher Erkrankungen - in der Praxis gestalten könnte, lässt sich jedoch erst durch weitere umfangreichere Untersuchungen dieser Art feststellen. Sie fordern für komplexe invitro-Modelle dieser Art zunächst eine grössere Anzahl an Blutspendern. Sollten sich die hier beobachteten Befunde dann verfizieren lassen, dürfte es darüber hinaus für zukünftige klinische Studien interessant sein, nach Verabreichung dieser beiden homöopathischen Komplexmittel nach ähnlichen immunologischen Effekten ex vivo zu suchen.

\section{Danksagung}

Diese Untersuchungen wurde von der Pharmazeutischen Fabrik Dr. Reckeweg \& Co $\mathrm{GmbH}$, Bensheim, finanziell unterstützt.

\section{Literatur}

[1] Wiesenauer M, Noll J, Häussler S: Verbreitung und Anwendungshäufigkeit von Naturheilverfahren. Therapeutikon 1997;2:93-96.

[2] Tuffs A: Three out of four Germans have used complementary or natural remedies. BMJ 2002;325(7371):990.

[3] Jobst KA: Homeopathy, Hahnemann, and The Lancet 250 years on: a case of the emperor's new clothes? J Altern Complement Med 2005;11(5):751-754.

[4] Gross H: Neue Studien sollen Vorwürfe der Wirkungslosigkeit entkräften. Wie stark sind die Argumente der Homöopathie? MMW Fortschr Med 2005; 147(49-50):10

[5] Degele N: Alternativmedizin zwischen Anpassung und Ausstieg: das Beispiel Homöopathie. Gesundheitswesen 2000;62(2):53-58.

[6] Weingärtner O: Homöopathische Potenzen, Springer Verlag Berlin, Heildelberg, New York 1992

[7] Weingärtner O: Modellbildung und homöpathische Potenzen, Forschende Komplementärmedizin 1997;3:136-140.

[8] Weingärtner O: What is the therapeutically active ingredient of homeopathic potencies? Homeopathy 2003; 92(3):145-151.

[9] Meyer-Wegener J: Homöopathische Potenzen und die Suche nach ihrer physikalischen Struktur, KVC Verlag Essen 2001.

[10] Paul AT, Gohil VM, Bhutani KK: Modulating TNF-alpha signaling with natural products. Drug Discov Today 2006;11(15-16):725-32.

[11] Heine $H$, Schmolz M: Immunologische Beistandsreaktion durch pflanzliche Extrakte in Antihomotoxischen Präparaten. Biologische Medizin 1998;29(1):12-14.

[12] Parkin J; Cohen B: An overview of the immune system. Lancet 2001;357(9270): 1777-1789.

[13] Feghali CA, Wright TM: Cytokines in acute and chronic inflammation. Front Biosciences 1997;2:2-26.

[14] Meyaard L, Hovenkamp E, Pakker N et al.: Interleukin-12 (IL-12) production in whole-blood cultures from human immunodeficiency virus-infected individuals studied in relation to
IL-10 and prostaglandin E2 production. Blood 1997;89(2): 570-576.

[15] Cree IA, Blair AL: Measurement of phagocyte chemiluminescence using microtitre plate luminometers. FEMS Microbiology Letters 1990;64:19-19.

[16] Prescott SL, Dunston JA, Hale J, Brecker L, Lehmann H, Weston S, Richmomd P: Clinical effects of probiotics are associated with increased interferon- $\gamma$ responses in very young children with atopic dermatitis. Clin Exp Allergy 2005;35:1555-1564.

[17] Schaub B, Bellou A, Gibbons FK, Velasco G et al.: TLR-2 and TLR-4 stimulation differentially induce cytokine secretion in human neonatal, adult and murine mononuclear cells. J Interferon Cytokine Res 2004; 24(9):543-552.

[18] Gordon S: Alternative activation of macrophages. Nature Reviews/lmmunol 2003;3 23-35.

[19] Fiorentino DF: IL-10 inhibits cytokine production by activated macrophages. J Immunol 2000;147(11):3815-3822.

[20] Borish L, Rosenwasser LJ: Update on cytokines. J Allergy Clin Immunol 1996;97(3): 719-733.

[21] Heine H: Grundlagen der Regulationsmedizin. Ärztezeitschrift für Naturheilverfahren 2000;41(2):82-93

[22] Schmolz M, Heine H: Homöopathische Substanzen aus der Antihomotoxischen Medizin modulieren die Synthese von TGF- $\beta 1$ in menschlichen Vollblutkulturen. Biologische Medizin 2001;30(2):61-65.

[23] Schmolz M, Weiser M: Wirkungen eines Antihomotoxikums auf das Immunsystem des Menschen. Biologische Medizin 2001; 30(3):132-135.

[24] O'Garra T: T-cell differentiation. Commitment factors for T-helper cells. Curr Biology 2000; 10:R492-R494.

\section{Korrespondenzadresse:}

Dr. Manfred W. Schmolz

EDI (Experimental and Diagnostic Immunology) $\mathrm{GmbH}$ Aspenhaustrasse 25, DE-72770 Reutlingen info@edigmbh.de 